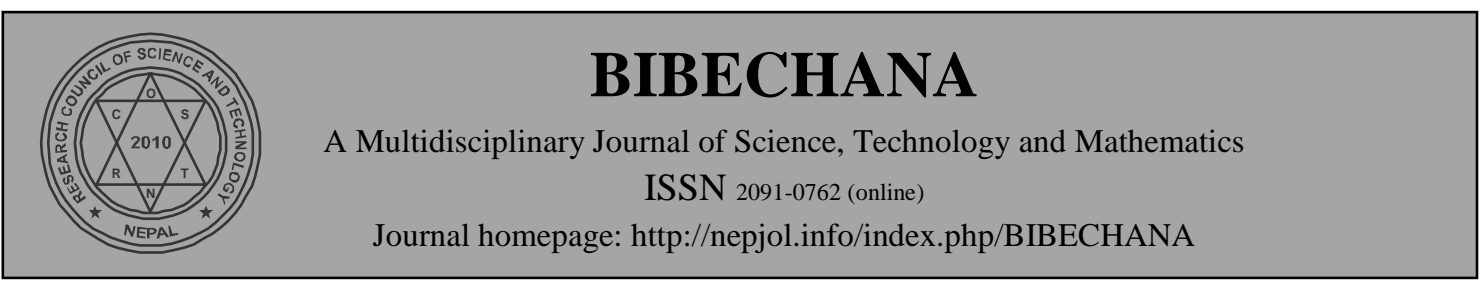

\title{
Characterization of Frechet spaces with nuclear Kothe quotients
}

\author{
Satya Narayan Sah \\ Department of Mathematics \\ M.M.A.M. Campus, T.U., Biratnagar, Nepal \\ E-mail: satyanarayansah33@yahoo.com \\ Accepted for publication: February 02, 2014
}

\begin{abstract}
In this paper our result based on the characterization of Frechet spaces with nuclear Kothe quotients is in terms of the following condition which labeled as $\star$. We show that a Frechet spaces E satisfies condition $\star$ if and only if it has a quotient which admits continuous norm and satisfies condition $\circledast$. For the condition $\circledast$, there exists $\ell$ such that for every $k$ there exists $j$ such that the $\|.\|_{k}$ closure of $E_{\ell}^{\prime}$ is not closed in $E_{j}^{\prime}$.
\end{abstract}

(C) 2014 RCOST: All rights reserved

Keywords: Frechet spaces; Kothe spaces; Quotient.

\section{Introduction}

Frechet spaces have played an important role in functional analysis from its very beginning. Many linear spaces of holomorfic, differentiable or continuous functions which arise in connection with various problems in analysis and its applications are defined by (at most) countably many conditions, whence they carry a natural Frechet topology (if they are, in addition, complete). C. Bessaga and A. Pelczynski showed that a Frechet space fails to admit a continuous norm if it has a subspace isomorphic to $\omega$ in 1957 [1]. If a Frechet space admits a continuous norm then so does every subspace, which simplifies the problem a little. Again, in 1959, Bessaga, Pelczynski and S. Rolewicz showed that a Frechet space which admits continuous norm has a nuclear Kothe subspace iff it is not Banach [2]. Thus it follows that, in general, a Frechet space has a nuclear Kothe subspace iff it has a non-Banach subspace which admits continuous norm. If we consider only nuclear Frechet spaces, then we are looking for Kothe quotients and here the problem has a positive solution: every nuclear Frechet space not isomorphic to $\omega$ has a Kothe quotient. The proof is given in [3].

In the present paper we find some characterization of the Frechet spaces with nuclear Kothe quotients. In view of the open mapping theorem the condition $\square$ has the following equivalent formulation :

$$
\exists \mathrm{l} \ni \forall \mathrm{k} \exists \mathrm{j} \ni \sup \{\|.\| ' \mathrm{k}: \mathrm{u} \in \mathrm{El} \text { ', }\|.\| \mathrm{j} \leq 1\}=\infty
$$

In this form our condition is very close to being a dual to the following condition used by Bessaga, Pelczynski and Rolewicz [4] in thier determination of those Frechet spaces which have nuclear Kothe subspaces:

$$
\forall \mathrm{k} \exists \mathrm{j} \ni \sup \{\|\mathrm{x}\| \mathrm{j}: \mathrm{x} \in \mathrm{Y},\|\mathrm{x}\| \mathrm{k} \leq 1\}=\infty
$$

for every subspace $y$ of $\mathrm{E}$ with finite co-dimension. 
It is easy to check that this condition $\square$ is independent of the choice of $(\|\cdot\| \mathrm{k})$.

For definitions and notations we refer to the book of G. Kothe [5]. Some of them which are used in this paper are following:

\section{Notations}

$E^{\prime}$ - the dual of $E$ will be considered to have the strong topology from $E$

$\mathrm{E}^{*}$ - the completion of $\mathrm{E}$

$E^{\prime \prime}-$ the dual of $E^{\prime}$

$\mathrm{A} 0$ - the polar in $\mathrm{E}$ or $\mathrm{E}^{\prime}$ where $\mathrm{A} \subseteq \mathrm{E}$ or $\mathrm{A} \subseteq \mathrm{E}^{\prime}$

$\|\cdot\| \mathrm{k}-\quad$ the semi-norm on $\mathrm{E}$, where $\mathrm{K}=1,2,3, \ldots \ldots$.

$\|\cdot\| \cdot k$ - the dual norm on $\mathrm{E}^{\prime}$

$(\|\cdot\| \mathrm{k})$ - the fundamental sequence of semi-norms

$E^{\prime} k$ - the Banach space determined by the unit ball of the dual norm $\|\cdot\|^{\prime} k$ on $E^{\prime}$, where $\mathrm{k} \in \mathrm{N}$

\section{Definitions}

Definition 3.1. A Frechet space is a metrizable, complete cocally convex vector space [6].

\# Its topology is defined by an increasing sequence of semi- norms $(\|\cdot\|) \mathrm{k}$ called a fundamental sequence of semi-norms. If one of these semi-norms is a norm we say that the space admits continuous norm.

\# A metrizable topological vector space (TVS) is complete if every Cauchy sequence is convergent $[5,7]$.

Definition 3.2. Nuclear kothe spaces are those Frechet spaces which have quotients that are nuclear, admit continuous norm and have a basis.

Definition 3.3. Let a linear subspace $M$ of a linear space $E$, consider the sets

$$
\mathrm{X}(\mathrm{M})=\mathrm{X}+\mathrm{M}=\{\mathrm{X}+\mathrm{Y}: \mathrm{Y} \in \mathrm{M}\}
$$

for each element $\mathrm{X} \in \mathrm{E}$. The collection of these so called equivalence classes becomes a linear space E/M, the quotient space of $\mathrm{E}$ by $\mathrm{M}$ if

and

$$
\begin{aligned}
& X(M)+Y(M)=X+Y+M=\{X+Y+Z: Z \in M\} \\
& \alpha X(M)=\alpha X+M=\{\alpha X+Z: Z \in M\}[8,9] .
\end{aligned}
$$

\section{Theorems}

\section{Theorem 4.1. (Open Mapping Theorem)}

If $\mathrm{E}$ and $\mathrm{F}$ are Frechet Spaces, $\mathrm{A}: \mathrm{E} \rightarrow \mathrm{F}$ linear, continuous and surjective, then $\mathrm{A}$ is open [6].

\section{Theorem 4.2. (Bipolar Theorem)}

If $\mathrm{E}$ is a locally convex and $\mathrm{M} \subset \mathrm{E}$ absolutely convex then $\mathrm{M}^{00}=\mathrm{M}^{-}[6]$.

\section{Theorem 4.3.}

If $\mathrm{E}$ is a Frechet Space and $\mathrm{M} \subset \mathrm{E}$ a closed subspace then $\mathrm{M}$ and $\mathrm{E} / \mathrm{M}$ are Frechet Spaces.

\section{Corollary 4.4.}

If $E$ is a Frechet Space then $E^{\prime}$ is a complete locally convex space which has a countable fundamental system of bounded sets. 


\section{Corollary 4.5.}

If $\mathrm{E}$ is isomorphic to a countable product of Banach Spaces then $\mathrm{E}$ does not have a nuclear Kothe quotient.

\section{Corollary 4.6.}

Every Frechet Montel Space not isomorphic to $\omega$ has a nuclear Kothe quotient.

\section{Proof:}

If $\mathrm{E}$ is a Frechet Montel Space then $\mathrm{E}$ is separable and reflexive. We will prove that $\mathrm{E}$ is not a quojection. Let $E$ be the projective limit of the surjections $A_{K}$ : $E_{K+1} \rightarrow E_{K}$. We may assume that $A_{K}$ maps the unit ball of $E_{K+1}$ on to the unit ball of $E_{K}$. Also, since $E$ is not isomorphic to $\omega$ we may assume that $E_{1}$ is infinite dimensional. Then, viewing $\mathrm{E}$ as the projective limit, it is easy to see that $\left\{\left(\mathrm{x}_{\mathrm{k}}\right) \in \mathrm{E}: \mathrm{x}_{\mathrm{k}}\right.$ is in the unit ball of $\left.E_{k} \forall_{k}\right\}$ is closed, bounded subset of $E$. But the projection of this set in $E_{1}$ is the unit ball so it is not compact [10,11]. Hence the set is not compact in $\mathrm{E}$ which is a contradiction.

We are now ready for the main result of this paper.

\section{Proposition 4.6.}

A Frechet space E satisfies condition $\circledast$ iff it has a quotient which admits continuous norm and satisfies condition $\circledast$.

\section{Proof:}

Suppose that the Frechet space E satisfies condition - . We may take $\ell=1$ and $j=k+1$ for this condition. Let $\mathrm{M}=\left(E_{l}^{s}\right)^{\circ \circ}$ which, by the bipolar theorem, is the closure of in $E_{l}^{s}$ the weak topology from E. Now we prove that $\mathrm{E} / \mathrm{M}^{\circ}$ is the desired quotient.

At first, we check that $\mathrm{E} / \mathrm{M}^{\circ}$ admits continuous norm. For this, let $\mathrm{x} \in \mathrm{E}$ and assume that the seminorm in $\mathrm{E} / \mathrm{M}^{\circ}$ induced by $\|\cdot\| 1$, annihilates $\mathrm{x}+\mathrm{M}^{\circ}$. This means that

$$
\exists(\mathrm{yn}) \subset \mathrm{M}^{\circ} \ni \operatorname{limn}\|\mathrm{x}-\mathrm{yn}\| 1=0 .
$$

Let $\mathrm{u} \in \mathrm{M}$. Then, $\exists \cdot \epsilon E_{\varepsilon}^{*} \ni|\mathrm{u}(\mathrm{x})-\mathrm{v}(\mathrm{x})| \leq 1$.

So we have

$$
|\mathrm{u}(\mathrm{x})| \leq|\mathrm{v}(\mathrm{x})|+|\mathrm{u}(\mathrm{x})-\mathrm{v}(\mathrm{x})| \leq\left|\mathrm{v}\left(\mathrm{x}+\mathrm{y}_{\mathrm{n}}\right)\right|+1 .
$$

Since $v \in E_{1}^{\prime}$ it follows that $\lim v(x+y k)=o$, so $|u(x)| \leq 1$. This shows $x \in M^{\circ}$, so the semi-norm induced by $\|\cdot\|_{1}$, is a norm.

Next, we verify condition $\circledast$ for $E / \mathrm{M}^{\circ}$. We fix $\mathrm{k}$ and let $\mathrm{U}_{\mathrm{k}}$ be the unit ball of $\|\cdot\|_{\mathrm{k}}$ in $\mathrm{E}$. Since $\mathrm{E}$ satisfies condition $\circledast$, we have a sequence $\left(\mathrm{u}_{\mathrm{n}}\right) \subset \mathrm{E}_{1}^{\prime}$ with $\|\mathrm{un}\|_{\mathrm{k}}^{\prime}>1$ and $\|\mathrm{un}\|_{\mathrm{k}+1}^{\prime} \leq \frac{1}{\mathrm{n}}$. This implies that, $\mathrm{u}_{\mathrm{n}} \in \frac{1}{\mathrm{n}} \mathrm{U}_{\mathrm{k}+1}^{\mathrm{O}}$ $\sim \mathrm{U}_{\mathrm{k}}^{\mathrm{o}}$ so a fortiori $\mathrm{u}_{\mathrm{n}} \notin\left(\mathrm{U}_{\mathrm{k}}+\mathrm{M}^{\circ}\right)^{\circ}$. Moreover, if $\mathrm{x} \in \mathrm{U}_{\mathrm{k}+1}, \mathrm{y} \in \mathrm{M}^{\circ}$, then as $\mathrm{u}_{\mathrm{n}} \in \mathrm{M}$, we have

$$
|\operatorname{nun}(\mathrm{x}+\mathrm{y})|=|\operatorname{nun}(\mathrm{x})| \leq 1 \text {. }
$$

Hence $u_{n} \in \frac{1}{n}\left(U k+1+M^{\circ}\right)^{\circ}$. Thus we have proved that un $\epsilon \frac{1}{n}\left(U k+1+M^{\circ}\right)^{\circ} \sim\left(U k+M^{\circ}\right)^{\circ}$. But, $\left(E / M^{\circ}\right)^{\prime}=$ $\mathrm{M}$ and the unit ball of the dual norm of the norm in $\mathrm{E} / \mathrm{M}^{\circ}$ induced by $\|\cdot\| \mathrm{k}$ is $\left(\mathrm{Uk}+\mathrm{M}^{\circ}\right)^{\circ}$. This proves that $\mathrm{E} / \mathrm{M}^{\circ}$ satisfies condition $\circledast$.

Now, conversely assume that $\mathrm{E} / \mathrm{F}$ be a quotient of $\mathrm{E}$. If (Uk) is a fundamental sequence of nbds. of 0 for $E$ then by general duality, (E/F)' can be represented as a vector subspace of $E^{\prime}$ and a fundamental 
sequence of equicontinuous sets for $(\mathrm{E} / \mathrm{F})^{\prime}$ is given by $\left(\mathrm{U}_{\mathrm{l}}^{\mathrm{O}} \cap+-\mathrm{F}^{\circ}\right) \mathrm{K}$. So if E/F satisfies condition $\circledast$ (say with $\mathrm{l}=1$ and $\mathrm{j}=\mathrm{k}+1)$ then $\exists\left(\mathrm{u}_{\mathrm{n}}\right) \subset \mathrm{F}^{\circ}$ and a sequence of constants $\left(\mathrm{C}_{\mathrm{n}}\right)$ with $\mathrm{u}_{\mathrm{n}} \in\left(\mathrm{C}_{\mathrm{n}} \mathrm{U}_{\mathrm{l}}^{\mathrm{O}} \cap \mathrm{F}^{\circ}\right) \cap\left(\frac{\mathbf{1}}{n z}\right.$ $\left.\mathrm{U}_{\mathrm{k}+1}^{\mathrm{O}} \cap \mathrm{F}^{\circ}\right) \operatorname{cs}\left(\mathrm{U}_{\mathrm{l}}^{\mathrm{O}} \cap \mathrm{F}^{\circ}\right)$. It follows that un $\epsilon \mathrm{E}_{\mathrm{l}}^{\prime}$, $\|\mathrm{un}\|_{\mathrm{k}+1}^{\prime} \leq \frac{1}{\pi}$ but since un $\epsilon \mathrm{F}^{\circ}$ then $\mathrm{u}_{\mathrm{n}} \notin \mathrm{U}_{\mathrm{l}}^{\mathrm{O}}$; so $\left\|\mathrm{u}_{\mathrm{n}}\right\|_{\mathrm{k}}^{\prime}$ $>1$. Therefore, E satisfies condition $\circledast$.

\section{Conclusion}

A Frechet space which admits continuous norm has a nuclear Kothe subspace if and only if it is not Banach. Thus it follows that, in general, a Frechet space has a nuclear Kothe subspace iff it has a nonBanach subspace which admits continuous norm.

\section{References}

[1] C. Bessaga, A. Pelczynski, Bull. Acad. Polon. Sei. Cl. Ill, 4 (1957) 375.

[2] C. Bessaga, A. Pelczynski, S. Rolewicz, Bull. Acad. Polon. Sei., 9 (1961) 677.

[3] G.L. Litvinov, Nuclear Space, Springer-Verlag, 2001.

[4] C. Bessaga and Ed Dubinsky, Arch Math., 31 (1978) 597.

[5] G. Kothe, Topological Vector Space I, Spiringer-Verlag, 1969.

[6] D. Vogat, Lectures on Frechet Spaces, Bergische Universitat Wuppertal, Sommer Semester, 2000.

[7] A.E.Taylor, Introduction to Functional Analysis, John Willey and Sons, Inc., London, 1961.

[8] A. Pietsch, Nuclear Locally Convex Space, Springer-Verlag Berlin Heidelberg, New York,1972.

[9] A.P. Robertson, W. Robertson, Topological Vector Spaces, Cambridge University Press, 1964.

[10] Ed Dubinsky, Springer-Verlag, Berlin and New York, 720 (1979) 123.

[11] V.B. Moscatelli, Bull. London Math. Soc., 12 (1980) 63.

[12] L. Holmstrom, Journal of Functional Analysis, 348 (1982) 12.

[13] Ed. Dubinsky, W. Robinson, Studia Math, 63 (1978) 267.

[14] W. Sliwa, Trans. Amer. Math. Soc., 362 (2010) 3273.

[15] W. Sliwa, Bull. Belg. Math. Soc. Simon Stevin, 14 (2007) 1017. 